\title{
Trans-subclavian approach for transcatheter aortic valve replacement
}

\author{
Daniel Richard Watson, Steven Joseph Yakubov, Jefferson Morton Lyons, Carlos Enrique Sanchez \\ Soto, Nathan Howard Kander, Anthony Theodore Chapekis, Arash Arshi, Aref Mahmoud Abou-Amro, \\ Geoffrey B. Blossom
}

Riverside Methodist Hospital, Columbus, OH, USA

Correspondence to: Daniel Richard Watson, MD. Riverside Methodist Hospital, Columbus, OH, USA. Email: Daniel.Watson@ohiohealth.com.

Submitted Jul 13, 2017. Accepted for publication Aug 17, 2017.

doi: 10.21037/acs.2017.09.07

View this article at: http://dx.doi.org/10.21037/acs.2017.09.07

\section{Clinical vignette}

We present an 84-year-old female with multiple comorbidities who is debilitated by recurrent admissions for congestive heart failure secondary to critical aortic stenosis. She reports a past medical history significant for chronic kidney disease, polymyalgia rheumatica, hypertension, recent pacemaker implantation and right hip replacement in the last 12 months. She reports that 2 weeks ago she was hospitalized for the third time in 3 months with "too much fluid on board". At the time she was told that her aortic valve was severely diseased, but she was not a surgical candidate due to the aforementioned risk factors. Since that admission, she reports progressive symptoms of fatigue and inability to walk to her mailbox. She presents to our Structural Heart Clinic for potential treatment of her aortic valvular disease.

Her cardiac echo revealed severe aortic leaflet calcification with decreased systolic excursion of her aortic valve cusps. The mean gradient across the valve was $45 \mathrm{mmHg}$ and the peak velocity was $4.38 \mathrm{~m} / \mathrm{s}$. Her aortic valve area was calculated at $0.56 \mathrm{~cm}^{2}$. She was deemed extreme risk for conventional open heart surgery for aortic valve replacement due to the previously described medical conditions, as well as her challenging stature, which included a height of 5 foot 2 inches tall and a weight of 345 pounds with a very large panniculus.

She thus underwent workup for transcatheter aortic valve replacement (TAVR). This included a CT scan of her entire body as well as a cardiac catheterization via her right radial artery. The catheterization revealed no inherent coronary artery disease but the CAT scan confirmed the significant challenges for access and completion of the transcatheter valve procedure. There was a $16 \mathrm{~cm}$ distance between the skin and her femoral artery for access purposes. She also had severe tortuosity of her aortic iliac segment with a bilateral hairpin turn from the common iliac arteries into the abdominal aorta. Additionally, her ascending aorta was very horizontal with the measurement of almost $60^{\circ}$. Assessment of alternative access for the procedure revealed that her subclavian artery was suitable for this procedure in that the mean diameter was $6.5 \mathrm{~mm}$. We thus chose this mode for access due to her multiple medical comorbidities, large body habitus, deep femoral arteries, tortuous vascular anatomy, rehabilitation challenge due to recent right hip replacement, and horizontal ascending aorta.

TAVR has emerged as a therapeutic option for the inoperable and high-risk patient since the first successful clinical implantation in 2002 (1). Potential access sites for TAVR are the femoral or subclavian arteries, the apex of the heart, or the ascending aorta (2). Percutaneous trans-femoral TAVR is the first choice for most groups and considered the least invasive option. However, there remains a considerable number of patients who are not candidates for this approach because of challenging body habitus, poor vascular access, tortuosity of the aorta or previous arterial surgical interventions. Further, transapical or trans-aortic approaches represent a formidable clinical footprint and challenging recovery for patients with frailty, suboptimal pulmonary function, or chest pathology (3).

The subclavian artery is regarded as a viable alternative access route for TAVR in these circumstances. In addition, the shorter distance between the access site and the aortic valve is even seen as a potential technical advantage because 
of the short distance between the delivery catheter and the annulus (4).

This technique report describes our initial clinical experience in over 200 patients using a retrograde approach of TAVR by direct delivery via the subclavian artery in patients who had poor femoral vascular access, challenging body habitus, poor respiratory function, and/or chest pathology that precluded consideration for the options discussed previously.

The use of the axillary and subclavian artery is familiar to cardiac surgeons. It has been advocated for routine use in cannulation for cardiopulmonary bypass (CPB) during thoracic aortic surgery as well as cases where conventional ascending aortic cannulation is contra-indicated. Its use as an alternative entry point to the vasculature is therefore appealing in endovascular procedures (5).

The ${ }^{18} \mathrm{~F}$ Medtronic CoreValve delivery system requires a minimum vessel diameter of $6 \mathrm{~mm}$. The ${ }^{14} \mathrm{~F}$ Medtronic Evolut R delivery system requires a minimum vessel diameter of $5.5 \mathrm{~mm}$. The ${ }^{14} \mathrm{~F}$ Edwards Sapien delivery system requires a minimum vessel diameter of $5.5 \mathrm{~mm}$ for sheath introduction, but must show potential vessel accommodation up to $8.1 \mathrm{~mm}$ for passage of the valve system through the sheath (6). Subclavian access was considered where transfemoral access was not feasible and provided that, the vessel met the minimum diameter requirements, showed no severe kinking, and no significant calcifications were present at the origin of the aortic arch.

\section{Operative technique-subclavian implantation}

The CoreValve Re-valving or Evolut $\mathrm{R}$ system was used in all patients except 7 . All cases were performed under general anesthesia until December of 2015 , when a conscious sedation approach was adopted. Under this policy, if anesthesia is comfortable with the patient's airway and potential for emergent intubation, local anesthesia is utilized for subclavian exposure with the assistance of intravenous Precedex. Central venous line, right radial artery line and a urinary catheter are inserted. The patients receive continuous cardiac monitoring and trans-esophageal echocardiography (TEE) when intubated. TTE is utilized for the conscious-sedation patients. The antibiotic prophylaxis follows the local protocol used for conventional surgery, administered 30 minutes prior to the skin incision. Temporary trans-venous right ventricular pacing wires are inserted through the right internal jugular vein. The patient is prepped and draped as per conventional surgery. A 6 Fr sheath was placed percutaneously via femoral or right radial arterial access, depending on the clinical scenario, and a pigtail catheter positioned in the non-coronary sinus. The proximal left axillary artery is exposed through a small, $3 \mathrm{~cm}$ infra-clavicular incision and encircled with soft rubber vessel loops. Although the surgical cut down to the subclavian artery is conducted in close proximity to the brachial plexus, nerve damage can be avoided by meticulous surgical technique typically involving cephalad mobilization. We had no incidence of nerve palsy in our series. Further, previous implantation of a permanent pacemaker at the site of access should be not be regarded as a contraindication because of concern for damaging the pacemaker device or wires. The desired access path is well superior and lateral to the entry of the leads into the venous system.

Following the administration of heparin $(8,000$ 10,000 I.U.), a $6 \mathrm{Fr}$ sheath is inserted into the vessel and a Lunderquist wire is positioned in the apex of the left ventricle, using standard catheter-exchange techniques, crossing the aortic valve. The axillary artery bleeding is then controlled utilizing vessel loop traction and a transverse arteriotomy performed. For Evolut R procedures if the vessel diameter is $>6 \mathrm{~mm}$, an $18 \mathrm{Fr}$ Cook sheath is inserted directly into the vessel and delivered around the aortic arch into the distal ascending aorta. A Cook sheath is chosen, as the braided nature of the polymer lends itself well to prevent kinking as it passes over the first rib. It is important to "pre-bend" the sheath to assure success in following the appropriate contour. A Lundequist wire is imperative to assure seamless passage, as well. In all cases the sheath diameter was either slightly larger or perfectly matched the axillary artery diameter, and there was no need to snare the vessels. Alternatively, the Evolut R In-line sheath system can be utilized with similar success, if desired. For Sapien procedures, the 14 Fr E-sheath is utilized in all cases and delivered to the arch in a similar fashion. It is important to maintain the "pleat" of the E-sheath in a cephalic orientation to prevent kinking of the delivery path.

The subsequent implantation technique used for the prostheses has been well described. Briefly, the native valve is dilated using a balloon valvuloplasty during rapid ventricular pacing. The loaded valve/stent is then introduced and correct positioning achieved using both angiographic and echocardiographic guidance. The prosthesis is deployed, and the result is assessed with echocardiography, hemodynamic measurements, and/ or aortography. Following satisfactorily implantation, the device is removed and the artery is reconstructed with a 
running 6/0 polypropylene suture. The wound is closed in layers. An angiogram of the axillary artery is not routinely performed at the end of the procedure, unless flow appears questionable by physical examination or doppler interrogation.

The incision is well tolerated by patients and allows very early mobilization of the patient after implantation. Healing of this area is associated with satisfying cosmetic results. We have not seen a postoperative wound infection after subclavian implantation in our patient population.

\section{Conclusions}

We have successfully utilized subclavian access for $20 \%$ of our TAVR experience in patients with challenges for access via the traditional trans-femoral approach. Our outcomes demonstrate the safety of the subclavian access for TAVR and comparability with the trans-femoral route, as well. We have also recognized significant advantages related to the shorter distance between access vessel and aortic valve and simplicity of valve delivery. Further study will delineate significant advantages in clinical outcomes and help clarify when subclavian access will have an indication for selected patients, and attention to the simple guidelines above will ensure smooth procedural steps and favorable outcomes.

\section{Acknowledgements}

None.

Cite this article as: Watson DR, Yakubov SJ, Lyons JM, Soto CE, Kander NH, Chapekis AT, Arshi A, Abou-Amro AM, Blossom GB. Trans-subclavian approach for transcatheter aortic valve replacement. Ann Cardiothorac Surg 2017;6(5):565-567. doi: 10.21037/acs.2017.09.07

\section{Footnote}

Conflicts of Interest: The authors have no conflicts of interest to declare.

\section{References}

1. Leon MB, Smith CR, Mack M, et al. Transcatheter aorticvalve implantation for aortic stenosis in patients who cannot undergo surgery. N Engl J Med 2010;363:1597-607.

2. Van Mieghem NM, Tchetche D, Chieffo A, et al. Incidence, predictors, and implications of access site complications with transfemoral transcatheter aortic valve implantation. Am J Cardiol 2012;110:1361-7.

3. Toggweiler S, Leipsic J, Binder RK, et al. Management of vascular access in transcatheter aortic valve replacement: part 2: Vascular complications. JACC Cardiovasc Interv 2013;6:767-76.

4. Ciuca C, Tarantini G, Latib A, et al. Trans-subclavian versus transapical access for transcatheter aortic valve implantation: A multicenter study. Catheter Cardiovasc Interv 2016;87:332-8.

5. Petronio AS, De Carlo M, Giannini C, et al. Subclavian TAVI: more than an alternative access route. EuroIntervention 2013;9 Suppl:S33-7.

6. Koehler T, Buege M, Schleiting H, et al. Changes of the eSheath Outer Dimensions Used for Transfemoral Transcatheter Aortic Valve Replacement. Biomed Res Int 2015;2015:572681. 